\title{
Introduction by the Guest Editor: Recent advances in biology and treatment of multiple myeloma
}

\author{
Hirokazu Tanaka ${ }^{1}$
}

Received: 9 January 2020 / Accepted: 15 January 2020 / Published online: 27 January 2020

(c) Japanese Society of Hematology 2020

Remarkable advance has been achieved in multiple myeloma (MM) over the last two decades. Especially, the treatment paradigm in $\mathrm{MM}$ with the introduction of novel agents has resulted in improved survival and tolerability, as well as deeper responses including achieving a minimal /measurable residual disease (MRD)-negative state. However, the disease is still considered incurable and displays heterogeneity in individual clinical presentation, course and outcome. This heterogeneity has often been caused by variable genomic and molecular characteristics in the malignant clone [1,2], while the technologies enabling molecular profile analysis have been made in our understanding of the biology and pathogenesis of MM. These advances continue to bring about evolving strict definitions of disease and prognosis, and the treatment paradigm combining stem cell transplant, targeted therapies, and immune therapies.

In this special issue of Internal Journal of Hematology, I invited four experts in the field to write reviews on "Recent Advances in Biology and Treatment of Multiple Myeloma" in "Progress in Hematology". This special issue is expected to deepen our knowledge of these advances in MM.

Drs. Yusuke Furukawa and Jiro Kikuchi present a comprehensive and insightful review on molecular basis of clonal evolution in MM and summarize the current knowledge of the mechanisms behind the complex nature of the genomic architecture and clonal evolution in MM, aiming at the development of better treatment strategies. Drs. Furukawa and Kikuchi's review not only guides some of what has been accomplished in the care of patients with MM, but also outlines possible suitable agents based on the disease biology of MM, with the hope that this type of approach will be beneficial with both long survival and minimal toxicity.

Hirokazu Tanaka

htanaka@med.kindai.ac.jp

1 Department of Hematology and Rheumatology, Kindai University Faculty of Medicine, Osaka-sayama, Japan
The current strategy for newly diagnosed MM involves induction, consolidation, and maintenance therapy. Proteasome inhibitors and immunomodulatory drugs constitute backbone agents that significantly improve outcomes among patients with MM [3], while the addition of new treatment options and combinations has added complexity in treatment selection for patients with MM. Furthermore, clinical practice outside of the context of clinical trials is an evolving field and real-world data should be also considered in the formulation of useful guidelines in the clinical settings and in the design of future studies for MM [4]. Dr. Kenshi Suzuki, who has had a leading role in the recent, pioneering work on care of myeloma patients, takes a global view of the current management of myeloma patients. Dr. Suzuki especially summarizes ideal, future-oriented therapeutic approaches for transplant eligible and newly diagnosed patients, highlighting risk adapted strategies, that offer individualized treatment options and may ultimately provide the possibility of a cure for those patients.

In daily clinical practice, a lot of hematologists are now faced with challenges including, optimal treatment sequencing and changing treatment goals. Although emerging data suggest that MRD negativity is one of the most powerful prognostic factors for treatment outcomes in patients with $\mathrm{MM}$, the use of MRD to guide treatment decisions in MM has been still considered and controversial [5, 6]. Dr. Hiroyuki Takamatsu has reviewed the most recent updates on MRD monitoring protocols in Japan and introduced several ongoing and future clinical trials involving MRD testing. With the optimum tools and situation to detect MRD, it will be becoming clearer in future that having such information can make management decisions easier, especially when used in the context of a clinical situation.

The innovative developments in immuno-oncology have also permeated into the treatment strategy of MM, characterized by immune disruption that might contribute to disease progression. Emerging immunotherapeutic agents, 
such as antibody-based therapies, checkpoint inhibitors, and chimeric antigen receptor (CAR)-engineered T-cell therapies have been developed as a means of targeting immune evasion of myeloma cells [7]. Despite the successes on the development of B-cell maturation antigen (BCMA)-directed CAR T-cell therapy, there remain significant challenges associated with CAR T-cell therapy and essential research efforts are currently in progress to develop new targets and approaches. Dr. Naoki Hosen summarizes the state of art in the field of CAR T-cell therapy against MM, and present their effort to develop a new CAR T-cell therapy with greater focus on targeting a protein conformation that is preferentially detected in myeloma cells.

I am confident that this issue of Internal Journal of Hematology comprehends the essence of significant advances in MM and presents the data for their current practical utilization and future strategies that offer the possibility of a cure for patients with MM.

\section{References}

1. Bolli N, Maura F, Minvielle S, et al. Genomic patterns of progression in smoldering multiple myeloma. Nat Commun. 2018;9:3363.
2. Schürch CM, Rasche L, Frauenfeld L, et al. A review on tumor heterogeneity and evolution in multiple myeloma: pathological, radiological, molecular genetics, and clinical integration. Virchows Arch. 2019. https://doi.org/10.1007/s00428-019-02725-3.

3. Anderson KC. Progress and paradigms in multiple myeloma. Clin Cancer Res. 2016;22:5419-27.

4. Richardson PG, San Miguel JF, Moreau P, et al. Interpreting clinical trial data in multiple myeloma: translating findings to the realworld setting. Blood Cancer J. 2018;8:109.

5. Kumar S, Paiva B, Anderson KC, et al. International Myeloma Working Group consensus criteria for response and minimal residual disease assessment in multiple myeloma. Lancet Oncol. 2016;17:e346.

6. Romano A, Palumbo GA, Parrinello NL, et al. Minimal residual disease assessment within the bone marrow of multiple myeloma: a review of caveats, clinical significance and future perspectives. Front Oncol. 2019;9:699.

7. Bonello F, Mina R, Boccadoro M, et al. Therapeutic monoclonal antibodies and antibody products: current practices and development in multiple myeloma. Cancers. 2019;12:15.

Publisher's Note Springer Nature remains neutral with regard to jurisdictional claims in published maps and institutional affiliations. 